\title{
A reassessment of Kelmayisaurus petrolicus, a large theropod dinosaur from the Early Cretaceous of China
}

\author{
Stephen L. Brusatte, Roger B.J. Benson, and Xing Xu \\ Acta Palaeontologica Polonica 57 (1), 2012: 65-72 doi: http://dx.doi.org/10.4202/app.2010.0125
}

The Early Cretaceous fossil record of large-bodied theropods from Asia is poor, hindering comparison of Asian predatory dinosaur faunas with those from other continents. One of the few large Asian theropod specimens from this interval is a partial skull (maxilla and dentary) from the Lianmugin Formation (?Valanginian-Albian), the holotype of Kelmayisaurus petrolicus. Most authors have either considered this specimen as an indeterminate basal tetanuran or a nomen dubium. Weredescribe $K$. petrolicus and note that it possesses a single autapomorphy (a deep accessory groove on the lateral surface of the anterior dentary), as well as a unique combination of characters that differentiates it from other theropods, affirming its validity. A phylogenetic analysis recovers $K$. petrolicus as a basal carcharodontosaurid, which is supported by various features: very deep interdental plates (a carcharodontosaurid synapomorphy), fused interdental plates (present in carchardontosaurids and a limited number of other theropods), and the absence of diagnostic features of other clades of large-bodied theropods such as abelisaurids, megalosauroids, and coelurosaurs. As such, Kelmayisaurus is the second known carcharodontosaurid from Asia, and further evidence that this clade represented a global radiation of large-bodied predators during the Early-mid Cretaceous.

Key words: Dinosauria, Theropoda, Carcharodontosauridae, anatomy, Cretaceous, China.

Stephen L. Brusatte [sbrusatte@ amnh.org], Division of Paleontology, American Museum of Natural History, Central Park West at 79th St., New York, NY, 10024, USA and Department of Earth and Environmental Sciences, Columbia University, New York, NY, USA; Roger B.J. Benson [rbb27@cam.ac.uk], Department of Earth Sciences, University of Cambridge, Downing Street, Cambridge, CB2 3EQ, United Kingdom; Xing Xu [xingxu@vip.sina.com], Institute of Vertebrate Paleontology and Paleoanthropology, Chinese Academy of Sciences, P.O. Box 643, Beijing 100044, People's Republic of China. 
This is an open-access article distributed under the terms of the Creative Commons

Attribution License (for details please see creativecommons.org), which permits unrestricted use, distribution, and reproduction in any medium, provided the original author and source are credited.

FoF 